\title{
Evaluation of recombinant modified vaccinia Ankara virus-based rhesus cytomegalovirus vaccines in rhesus macaques
}

\author{
Yujuan Yue $\cdot$ Zhongde Wang $\cdot$ Kristina Abel $\cdot$ Jinliang Li $\cdot$ Lisa Strelow $\cdot$ \\ Angelo Mandarino • Meghan K. Eberhardt • Kimberli A. Schmidt • \\ Don J. Diamond · Peter A. Barry
}

Received: 23 October 2007 / Published online: 15 January 2008

(C) Springer-Verlag 2008

\begin{abstract}
A vaccine consisting of rhesus cytomegalovirus (RhCMV) pp65-2, gB and IE1 expressed via modified vaccinia Ankara (MVA) was evaluated in rhesus macaques with or without prior priming with expression plasmids for the same antigens. Following two MVA treatments, comparable levels of anti-gB, pp65-2 and neutralizing antibody responses, and pp65-2- and IE1-specific cellular immune responses were detected in both vaccinated groups. Similar reductions in plasma peak viral loads were observed in these groups compared to untreated controls. This study demonstrates the immunogenicity and protective efficacy of rMVA-based RhCMV subunit vaccines in a primate host
\end{abstract}

Y. Yue and Z. Wang are co-first authors.

Y. Yue $(\bowtie) \cdot$ L. Strelow · M. K. Eberhardt · K. A. Schmidt .

P. A. Barry

Center for Comparative Medicine,

University of California-Davis,

County Rd 98 \& Hutchison Dr., Davis, CA 95616, USA

e-mail: yyue@ucdavis.edu

Z. Wang $\cdot$ J. Li $\cdot$ A. Mandarino $\cdot$ D. J. Diamond

Laboratory of Vaccine Research,

Beckman Research Institute of the City of Hope,

Duarte, CA 91010, USA

K. Abel · P. A. Barry

California National Primate Research Center,

University of California, Davis, CA 95616, USA

P. A. Barry

Department of Pathology and Laboratory Medicine,

University of California, Davis, CA, USA

K. Abel

Department of Internal Medicine,

Division of Infectious Disease, School of Medicine,

University of California, Davis, CA, USA and warrants further investigation to improve the efficacy of subunit vaccines against CMV.

Keywords Cytomegalovirus vaccine $\cdot$ Rhesus macaques . Modified vaccinia Ankara virus

\section{Introduction}

Development of an effective vaccine for human cytomegalovirus (HCMV) has been designated a "level 1" priority by the Institute of Medicine because of the devastating impact of congenital infection by HCMV which can have lifelong consequences [19]. The strict species-specificity of the virus requires assessment of vaccine strategies in a cognate animal model. Rhesus CMV (RhCMV) infection of rhesus macaques (Macaca mulatta-Mmu) strongly recapitulates HCMV infection in humans in terms of the similarity of viral genome, persistence, pathogenesis and host immune responses [2-4, 26, 27], therefore, it represents a clinically relevant model to evaluate HCMV vaccine strategies.

Measurement of natural immunity to HCMV supports targeting the predominant neutralizing antibody response antigen $\mathrm{gB}$ and the strongly recognized cellular immunogens pp65 and IE1 as the optimal antigens in a vaccine formulated to prevent infection and control disease [28]. Previously, a study in Mmu demonstrated that immunization with DNA vaccines expressing RhCMV pp65-2 and truncated $\mathrm{gB}$ with deletion of transmembrane and intracellular domains $(\mathrm{gB} \Delta)$ stimulated antigen-specific $\mathrm{CD} 8+\mathrm{T}$ cell and antibody responses and provided limited protection against challenge [25]. A notable observation of this study was that a combination of DNA priming administered five times in 50 weeks followed by a heterologous boost (in this case, by the virus challenge) stimulated a rapid and profound 
increase in neutralizing antibody titers. In this report, we compared an approach in which recombinant modified vaccinia Ankara (rMVA) expressing RhCMV pp65-2, gB $\Delta$ and IE1 antigens was administered twice to Mmu versus an identical schedule of rMVA administration preceded by a single DNA prime. Our primary goal was to establish whether these sub-unit vaccines are effective for controlling an experimentally applied CMV infection and to test whether DNA priming enhances the effectiveness of rMVA to stimulate protective immunity in this model.

\section{Results}

\section{RhCMV-rMVA construction and expression}

As a first step, the genes encoding $\mathrm{RhCMV}_{68-1}$ pp65-2 and $\mathrm{gB} \Delta$ were amplified from previously described plasmid expression vectors [25, 27] and engineered into the pZWIIA MVA transfer vector (Rh-gB-pp65-pZWIIA, Fig. 1a), using established protocols [21]. Similarly, the RhCMV IE1 gene was amplified from a plasmid (pND/IE1) containing the cDNA (exons 1-4) for the $\mathrm{RhCMV}_{68-1}$ IE1 [11] and engineered into the pLW51 MVA transfer vector (Rh IE1-pLW51, [22]).

rMVA expressing either both RhCMV gB $\Delta$ and pp65-2 (Rh gB-pp65-MVA) or IE1 alone (Rh IE1-MVA) was generated on BHK-21 cells via homologous recombination.
The protein expression levels for RhCMV gB, pp65-2 and IE1 in infected BHK-21 cells were confirmed by Western blot using polyclonal antibodies to RhCMV gB, pp65-2, and IE1 by chemiluminescence detection (ECL, Amersham Pharmacia Biotech, Buckinghamshire, UK) (Fig. 1b).

Although the genetic stability of the rMVA constructs was not directly evaluated, previous work using the same recombination plasmid pZWIIA to express HCMV soluble gB and/ or pp65 and IE1 exon-4 demonstrated that the resultant rMVA constructs were stable through four passages [20,21]. The genetic instability of rMVA that has been observed usually happens when attempting to express certain integral membrane proteins at high levels, which may be due to the toxicity of over-expressed proteins [23]. The genetic stability of rMVA expressing membrane proteins can be enhanced after truncating the cytoplasmic domain [22, 23]. In this report, we used the same strategies and methods to construct the rMVAs expressing RhCMV truncated gB, pp65-2 and IE1. RhCMV gB, pp65-2 and IE1 are homologues of HCMV gB, pp65 and IE1 and the results in Fig. 1b confirm expression of the foreign inserts. Aliquots of the same rMVA stocks used to demonstrate expression in infected tissue culture cells (Fig. 1) were used to infect rhesus macaques.

\section{Vaccination-induced immune responses}

Six age-matched RhCMV-seronegative Mmu (2 years of age) were divided into three groups-DNA prime/rMVA/

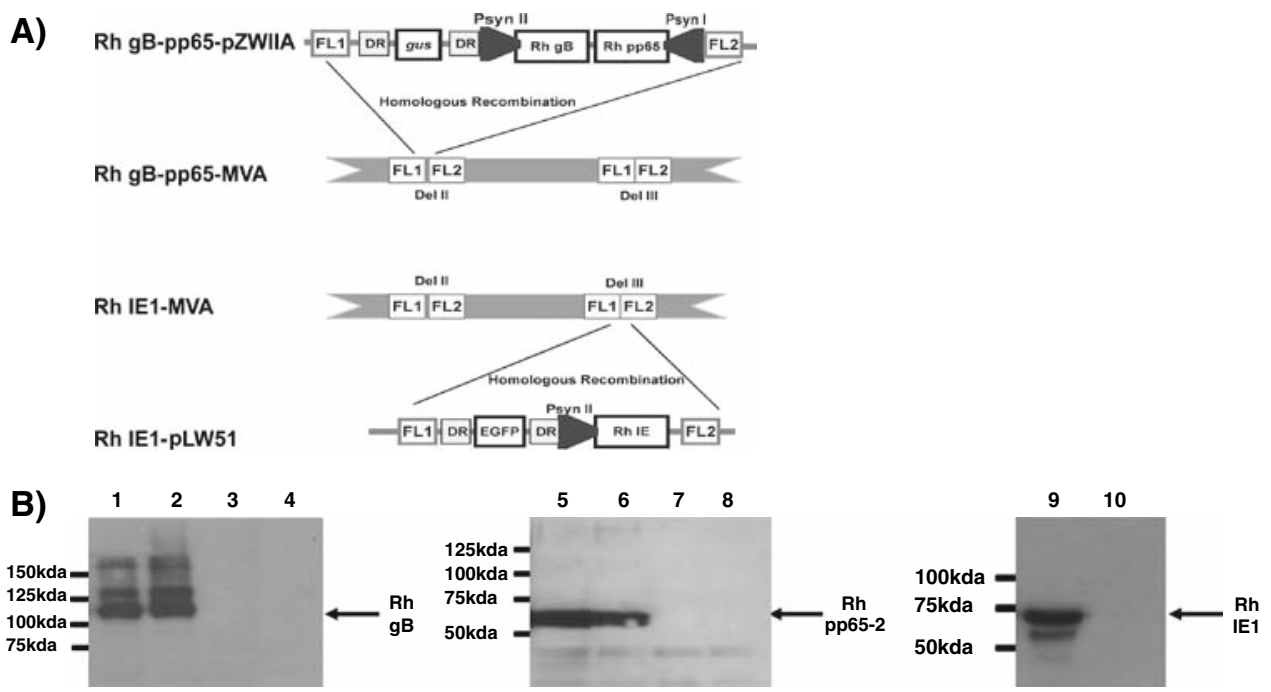

Fig. 1 rMVA construction and expression in vitro. a Construction of two rMVA constructs containing either truncated RhCMV $\mathrm{gB}$ and pp65-2 genes (Rh gB-pp65-MVA) or IE1 gene alone (Rh IE1-MVA). FL1 and FL2 represent distinct flanking MVA DNA sequences abutting either deletions II (Del II) or III (Del III). DRs are identical direct repeat sequences used for intragenomic homologous recombination to remove the marker genes, gus and EGFP. The expression of RhCMV $\mathrm{gB}$, pp65-2 and IE are under the control of synthetic vaccinia promoters, Psyn I and II, respectively. Rh gB-pp65-pZWIIA and Rh IE1-
pLW51 are transfer plasmids. b Western blot detection of Rh gB, pp65-2 and IE1 protein from Rh gB-pp65-MVA and Rh IE1-MVA infected BHK-21 cells. Lanes 1 and 5 cell lysate of Rh gB-pp65-MVA \#1 infected BHK-21 cells; lanes 2 and 6 cell lysate of Rh gB-pp65MVA \#2 infected BHK-21 cells; lanes 3, 7 and 10 cell lysate of wild type MVA infected BHK-21 cells as (-) negative control; lanes 4 and 8 cell lysate from uninfected BHK-21 cells; lane 9 cell lysate of Rh IE1-MVA infected BHK-21 cells 
rMVA boost (DNA/rMVA), rMVA prime/rMVA boost (rMVA) and untreated control. Initially, DNA/rMVA animals were immunized intramuscularly and intradermally with a mixture of three plasmids (in pND vector [13]) expressing RhCMV pp65-2, gB $\Delta$, and IE1 at week 0, following a previously published protocol [25]. Subsequently, all the DNA/rMVA and rMVA animals were injected intramuscularly with a mixture of Rh gB-pp65-MVA and Rh IE1-MVA $\left(5 \times 10^{8}\right.$ pfu of each) at weeks 6 and 12 (Fig. 2). The elicited immune responses were measured by antigenspecific ELISA, an EGFP-fluorescence intensity reductionneutralizing assay using an EGFP-RhCMV [6], and intracellular cytokine staining (ICS). The ICS was performed as previously described protocols [5] to detect the frequency of CD4+ and CD8+ T cells that express IFN- $\gamma$ or TNF- $\alpha$ upon re-stimulation with a pool of overlapping peptides (15-mers overlapping by 11 amino acids) comprising full-length IE1 or pp65-2 antigen [26]. Briefly, freshly isolated $1.0 \times 10^{6}$ peripheral blood monocytes (PBMCs) were stimulated for $6 \mathrm{~h}$ with the peptide pool or medium in the presence of monoclonal antibodies to CD28 and CD49d. Brefeldin A was added to the culture for the final $5 \mathrm{~h}$ of stimulation. SEB stimulated PBMCs were used as positive control. The stimulated cells were first surface stained with conjugated antibodies to $\mathrm{CD} 3$ and $\mathrm{CD} 8$, or $\mathrm{CD} 3$ and $\mathrm{CD} 4$ for $30 \mathrm{~min}$ at $4^{\circ} \mathrm{C}$. After fixation and permeabilization, the cells were incubated with conjugated anti-cytokine monoclonal antibodies for $30 \mathrm{~min}$ at $4^{\circ} \mathrm{C}$. The stained cells were then washed, and resuspended in $1 \%$ paraformaldehyde. Three hundred thousand lymphocyte events were collected on a FACS ARIA instrument (Becton-Dickinson). Data were analyzed using Flowjo software (version 8.1, Treestar, Ashland, OR).

DNA/rMVA and rMVA animals exhibited different kinetics of antigen-specific and neutralizing antibody responses (Fig. 3). As expected, single DNA immunization was not effective by itself, but effectively served to prime the animals for rMVA boosting (0-6 weeks, Fig. 3). Following the first rMVA vaccination (6-12 weeks, Fig. 3), both DNA/
rMVA animals generated higher levels of anti-gB, pp65-2 and IE1 antibodies and 50\% neutralizing titers $\left(\mathrm{NT}_{50}, 527\right.$ and 108, respectively) than Mmu receiving rMVA without DNA priming (Fig. 3). The two Mmu, only receiving rMVA, had low but detectable anti-gB responses and only one exhibited demonstrable $\mathrm{NT}_{50}$ (Animal \#4: 55) (Fig. 3). Anti-gB and pp65-2 antibody levels and $\mathrm{NT}_{50}$ rose sharply in both groups after the second rMVA immunization such that there were no differences between the two vaccine groups (12-18 weeks, Fig. 3). For the DNA/rMVA animals, the second rMVA boost stimulated peak anti-gB and antipp65-2 antibody responses within 1 week to levels higher than those observed after the first boost at weeks 7-8, but not peak IE1 antibody and $\mathrm{NT}_{50}$ (185 and 77, respectively). In contrast, the $\mathrm{NT}_{50}$ in the rMVA group were appreciably higher than those observed after the first rMVA immunization (294 and 122, respectively). rMVA immunization alone did not induce detectable anti-IE1 antibodies (Fig. 3).

IE1- and pp65-2-specific CD4+ and CD8+ T cells were also detected in DNA/rMVA and rMVA animals with different kinetics and magnitudes (Fig. 4). DNA priming followed by rMVA boosting generated both pp65-2- and IE1-specific CD4+ and CD8+ T cells mostly at 1-2 weeks after the first MVA booster, when a positive response was defined as a $0.01 \%$ increase of each stained cell population compared to the pre-vaccination baseline. The second rMVA immunization only stimulated transient increases prior to challenge. For the rMVA group, IE1- and pp65-2specific CD4+ and CD8+T cell responses in both animals were frequently detectable after the second rMVA immunization with the peak responses at week 14 or 18 .

Plasma viral loads post-challenge

To evaluate the protective efficacy of the two vaccine strategies, all the animals were challenged intravenously (IV, $2.0 \times 10^{5} \mathrm{pfu}$ ) and subcutaneously (SC, $\left.6 \times 10^{5} \mathrm{pfu}\right)$ with $\mathrm{RhCMV}_{68-1} 6$ weeks after the second rMVA vaccination.

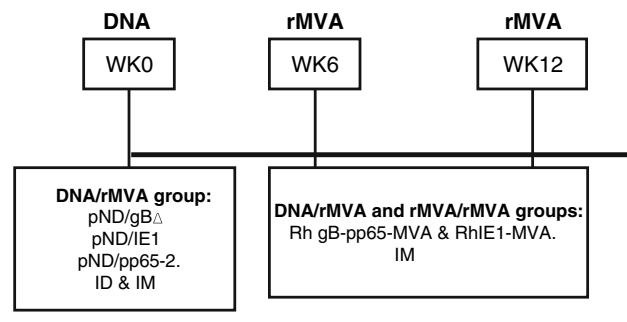

Fig. 2 DNA and rMVA vaccination regimen and RhCMV challenge. Six age-matched macaques were divided into three groups-DNA/rMVA, rMVA, and untreated control group. The DNA/rMVA animals were first primed with three DNA plasmids by intradermal (ID, $50 \mu \mathrm{g}$ of each plasmid) and intramuscular (IM, $150 \mu \mathrm{g}$ of each plasmid) needle injection at week 0 . At weeks 6 and 12, all of the DNA/rMVA and rMVA animals were immunized with rMVA vaccines $\left(5 \times 10^{8}\right.$ pfu of each rMVA vaccine). At week 18, all three groups of animals were intravenously (IV, $\left.2.0 \times 10^{5} \mathrm{pfu}\right)$ and subcutaneously (SC, $6.0 \times 10^{5} \mathrm{pfu}$ ) challenged with RhCMV strain 68-1. A quantitative real-time PCR (qPCR) was performed on the plasma samples collected prospectively from the time of challenge to measure RhCMV viral loads (RhCMV VL) 
Fig. 3 Antigen-specific antibody and neutralizing antibody responses in DNA/rMVA-, rMVA-vaccinated and control monkeys. Antibody responses to RhCMV gB, pp65-2 and IE1 were analyzed by antigen-specific ELISA. Absorbance units at $450 \mathrm{~nm}\left(\mathrm{~A}_{450}\right)$ were obtained at a dilution of 1:100 of plasma samples. 50\% neutralizing titers $\left(\mathrm{NT}_{50}\right)$ were titrated by an EGFP fluorescence reduction-neutralizing assay and were reported as the logarithmic values of the reciprocal endpoint dilution. The arrows indicate the time of DNA, rMVA and RhCMV administration and the horizontal dashed lines represent the threshold for a positive antibody response
Anti-gB Antibody
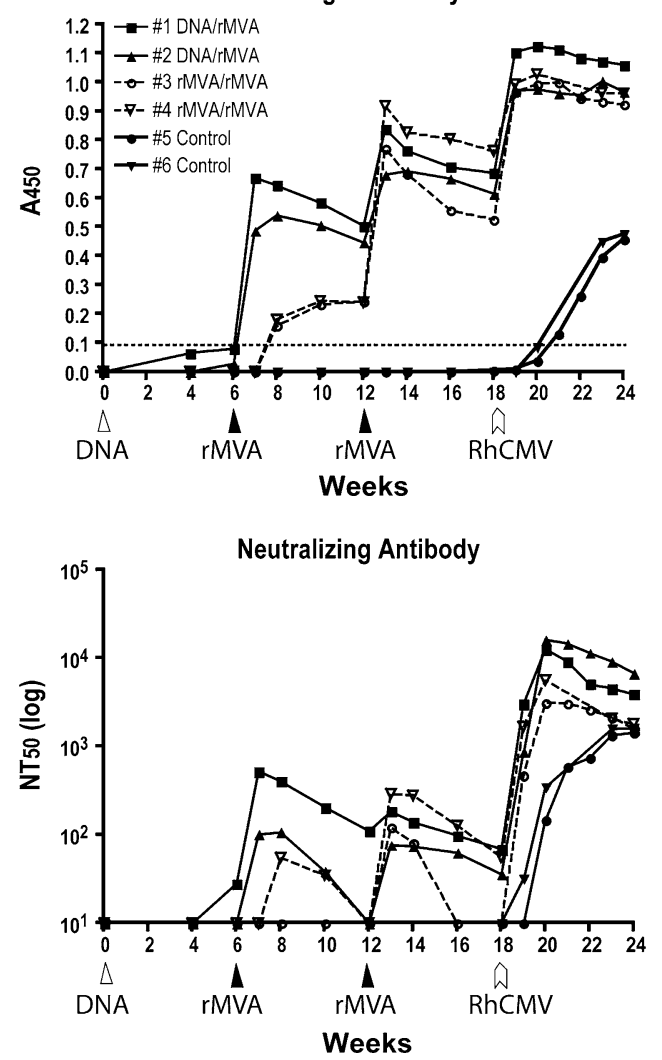

Anti-pp65-2 Antibody

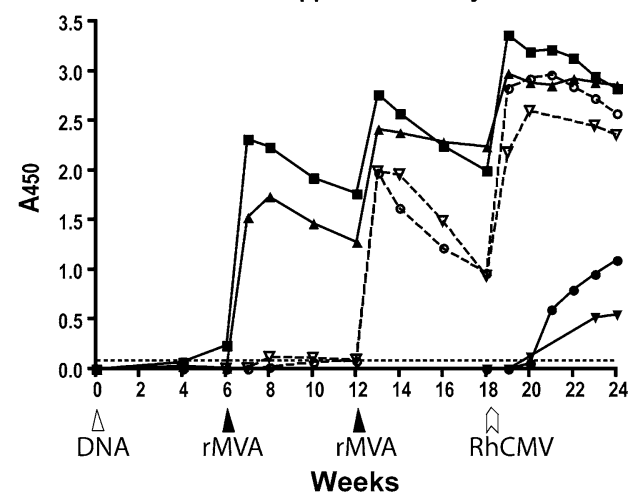

Anti-IE1 Antibody

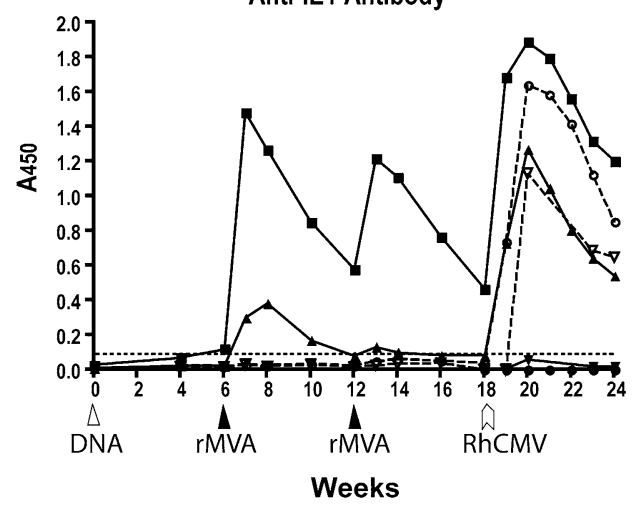

The plasma viral DNA loads were evaluated by real-time PCR [12] (Fig. 5a). The reduction levels of peak viral loads in vaccines were calculated as $(1-$ peak viral loads of vaccinee/peak viral loads of control animal) $\times 100 \%$. All four vaccinees exhibited different levels of reduction in peak plasma RhCMV genome copy numbers compared to the controls (Fig. 5b). One animal in each vaccine group (animals \#2 and \#3) had modest reductions (25.5-77.6\%), while the other animal in each group (animals \#1 and \#4) had profound reductions (90.8-98.9\%). Both of these latter animals were the same animals that had the higher $\mathrm{NT}_{50}$ titers at 1 week after the second rMVA immunization and the time of challenge. Although statistical tests did not show a significant correlation between the reduction levels of peak plasma viral loads and $\mathrm{NT}_{50}$ titers at 1 week after the second rMVA immunization (Spearman $r=0.80$, $P=0.33$ ) and at the time of challenge (Spearman $r=0.80$, $P=0.33$ ) in these four vaccinees, this may be due to the small numbers of Mmu. All four vaccinated animals initially cleared virus from plasma by day 10 , compared to days 13 and 17 for the two control monkeys (Fig. 5a). Transient detection of RhCMV in plasma (less than $3.5 \times 10^{3}$ copy number $/ \mathrm{ml}$ ) was noted for two of the vaccinees and one of the control animals (data not shown).

In addition to plasma viral loads, skin biopsies of two sites of RhCMV inoculation (SC) were obtained on days 7 and 10 post-challenge to evaluate RhCMV replication at local inoculation sites. IE1-expressing cells in skin tissue slides were analyzed by immunohistochemstry. No significant differences were observed between either the vaccinated and control groups (data not shown).

Immune responses post-challenge

All the vaccinees exhibited vigorous memory antibody responses post-challenge. These included anti-IE1 antibodies in the rMVA animals, which had no detectable antibodies prior to challenge (Fig. 3). The relative increases in $\mathrm{NT}_{50}$ of DNA/rMVA and rMVA animals at 1 and 2 weeks post challenge, compared to the time of challenge, were 24 to 44-fold and 101 to 453 -fold, respectively (Fig. 3). It was also noted that the $\mathrm{NT}_{50}$ of DNA/rMVA animals at 2 weeks post-challenge were approximately two- to fivefold above those of the rMVA animals. The normative range of $\mathrm{NT}_{50}$ in long-term RhCMV seropositive macaques is 231-3,348 ( $N=24$, mean 973, median 833) (unpublished data). DNA/ rMVA and rMVA/rMVA animals produced comparable $\mathrm{NT}_{50}$ at 1-week post-challenge and higher $\mathrm{NT}_{50}$ at 2-week post-challenge as compared to long-term naturally infected $\mathrm{Mmu}$, whereas the control animals had comparable $\mathrm{NT}_{50}$ after 4 weeks post-challenge. RhCMV challenge stimulated a rapid increase in the frequencies of IE1- and pp65-2-specific $\mathrm{CD} 8+\mathrm{T}$ cells in one of the DNA/rMVA treated $\mathrm{MMu}$ (animal \#2) and the frequency of pp65-2-specific CD8+ T 
Fig. 4 RhCMV pp65-2- and IE1-specific T cell responses in DNA/rMVA-, rMVA vaccinated and control monkeys. The intracellular cytokine staining was used to detect the frequency of CD4+ and CD8+ T cells that express IFN- $\gamma$ or TNF- $\alpha$ upon restimulation with a pool of overlapping peptides comprising full-length pp65-2 or IE1. The frequencies of IFN- $\gamma$ - and TNF$\alpha$-positive CD4+ and CD8+ T cells were shown after deducting the baseline frequencies at the pre-vaccination or challenge. The arrows indicate the time of DNA, rMVA and RhCMV administration
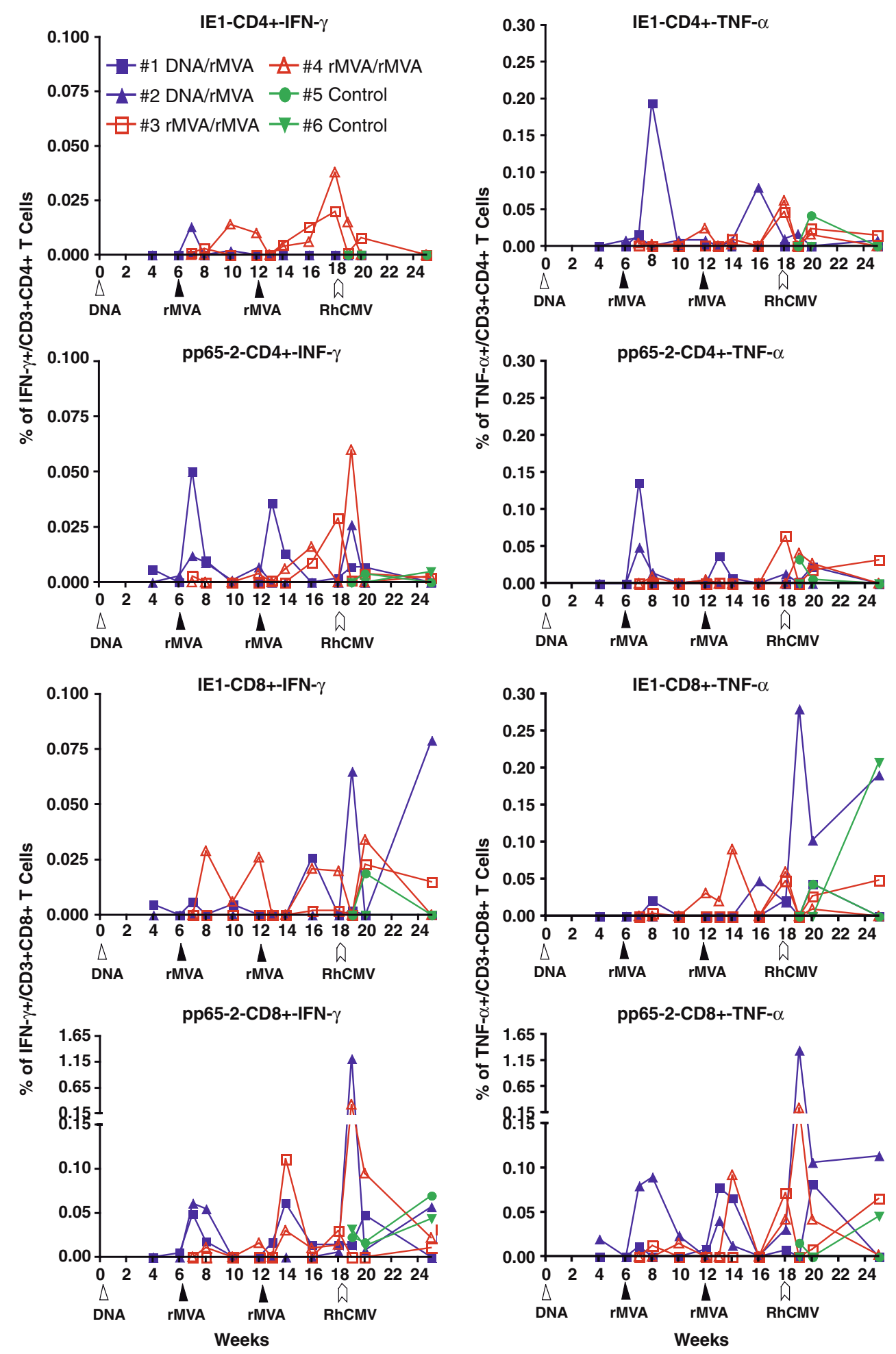

cells in one of the rMVA treated animals (animal \#4) at 1week post-challenge (Fig. 4). The frequencies were comparable to or higher than those observed in two unvaccinated naturally RhCMV-seropositive animals, suggesting a memory $\mathrm{T}$ cell response in these animals. One unvaccinated control produced a very high frequency of IE1-specific $\mathrm{CD} 8^{+} \mathrm{T}$ cells at 7 weeks of challenge.

\section{Discussion}

In this report, we demonstrate that rMVA, either given as the sole vaccine or part of a heterologous prime/boost, can result in a dramatic decrease in viral load after RhCMV challenge in the Mmu model. Noteworthy was the need for only a single application of DNA to effectively prime the 




B)



$\begin{array}{lllll}\text { Day of RhCMV challenge } & 69 & 36 & 0 & 56\end{array}$

Fig. 5 Plasma viral loads post-challenge. a The kinetics of plasma viral loads in DNA/rMVA-, rMVA-vaccinated and control animals. RhCMV genomic DNA in plasma was measured by a real-time PCR and was shown as RhCMV copy numbers per milliliter. b The reduction of peak viral loads in vaccinees (percentage) compared to the peak viral loads of control animals \#5 and \#6, respectively. The $\mathrm{NT}_{50}$ titers of each vaccinee at 1 week post-second rMVA and at the day of RhCMV challenge was shown at the bottom

immune system for a heterologous boosting to produce higher levels of immunogenicity than observed with three DNA applications without viral boosting [20].

Modified vaccinia Ankara has been established as a safe and potent antigen delivery system. Promising data obtained from both nonhuman primates and clinical trials have shown that a combination of DNA prime and rMVA boost is effective in stimulating effective cellular immune responses and providing high protective efficacy against HIV/SIV and malaria infection [7]. In this study, following the first rMVA boosting, the monkeys primed with only a single application of DNA plasmids elicited earlier and stronger antibody and cellular immune responses than those without priming. However, similar magnitudes of anti-gB, pp65-2 and neutralizing antibodies and IE1- and pp65-2specific CD4+ and CD8+ T cells were detected in both vaccinated groups after the second rMVA boost, with the exception of anti-IE1 antibodies, which were not detectable in rMVA animals.
Based on the level of IE1 expression in infected tissue culture cells (Fig. 1b), it was somewhat surprising that rMVA immunization in the absence of DNA priming did not stimulate detectable antibody responses. However, the results were not unprecedented. Other studies have shown that a recombinant canarypox expressing HCMV gB did not stimulate immune responses in vaccinated humans, despite expression both in vitro and in small animals (mice and guinea pigs) [1, 10]. Comparable to our study, the canarypox construct did prime the immune system as demonstrated by the memory response generated after infection with the live attenuated Towne vaccine. In our study, prior immunization with DNA or RhCMV infection post rMVA vaccination similarly demonstrated that RhCMV IE1 was recognized in vivo in the context of rMVA.

Both approaches provided comparable protection against challenge. It was also noted that the frequencies of pp65-2and IE1-specific CD4+ and CD8+ T cells in both vaccine groups generally were low, and strong memory $\mathrm{T}$ cell responses were only found in one animal in each vaccination group post-challenge. The reasons for the relatively low level of IE1- and pp65-2-specific T cell responses in this study are unknown. Other studies have demonstrated readily detectable levels of $\mathrm{T}$ cell responses to these $\mathrm{RhCMV}$ proteins in naturally infected or vaccinated Mmu $[25,26]$. Naturally infected Mmu with IE1 and pp65-2 responses were included as positive controls for all of the cellular immune assays. Future studies will address whether augmentation of vaccine-induced responses to IE1 and pp65-2 correlate with increased protection against RhCMV challenge.

An observation of interest in this study was the apparent influence of neutralizing antibody on the containment of viral challenge in which the levels of peak plasma viral load reduction were related to the levels of neutralizing antibodies prior to challenge. Although a larger numbers of Mmu are needed to confirm this observation, it further indicates the protective role of neutralizing antibodies against CMV $[14,17,18,24]$ and a gB-based vaccine strategy $[15,17$, 25]. Natural immunity studies have suggested that a vaccine that stimulates immunity equivalent to levels associated with natural infection could reduce the morbidity due to congenital CMV infection by $\sim 80 \%[8,9]$. While the employment of either DNA/rMVA or rMVA to deliver RhCMV gB did not induce a relevant natural level, longlived neutralizing antibody response, other approaches including co-administration of GM-CSF, an adjuvant for the induction of NT [16], either expressed via DNA or MVA are being explored.

In summary, this study for the first time demonstrates the capacity of rMVA-based CMV vaccines to induce CMVspecific antibody and cellular immune responses in $\mathrm{Mmu}$, and the protective efficacy in reducing plasma viral loads 
following challenge. The data highlight the potential of rMVA-based CMV vaccines for humans and warrant continuing investigation of the DNA/rMVA approach in a larger number of Mmu to further test the possibility that subunit vaccines can interrupt horizontal transmission.

Acknowledgments The authors wish to thank Linda Wyatt and Bernard Moss for MVA vectors and Dan Barouch for advice on primeboost strategies. This work was supported by NIH grants to PAB (AI063356), DJD (CA30206-Prj3, CA077544 and AI062496), the California National Primate Research Center (RR000169), and City of Hope Cancer Center (CA33572).

\section{References}

1. Adler SP, Plotkin SA, Gonczol E, Cadoz M, Meric C, Wang JB, Dellamonica P, Best AM, Zahradnik J, Pincus S, Berencsi K, Cox WI, Gyulai Z (1999) A canarypox vector expressing cytomegalovirus (CMV) glycoprotein B primes for antibody responses to a live attenuated CMV vaccine (Towne). J Infect Dis 180:843-846

2. Baroncelli S, Barry PA, Capitanio JP, Lerche NW, Otsyula M, Mendoza SP (1997) Cytomegalovirus and simian immunodeficiency virus coinfection: longitudinal study of antibody responses and disease progression. JAIDS 15:5-15

3. Barry PA, Chang WLW (2007) Primate betaherpesviruses. In: Arvin A, Campadielli G, Moore P, Mocarski E, Roizman B, Whitley R, Yamanishi K (eds) Human herpesviruses: biology, therapy and Immunoprophylaxis. Cambridge University Press, London

4. Barry PA, Lockridge KM, Salamat S, Tinling SP, Yue Y, Zhou SS, Gospe SM Jr, Britt WJ, Tarantal AF (2006) Nonhuman primate models of intrauterine cytomegalovirus infection. ILAR J 47:49-64

5. Buxbaum S, Kraus FB, Hahn A, Beck O, Kabartas B, Doerr HW, Ludwig B (2006) Flow cytometric analysis of virus-specific T lymphocytes: practicability of detection of HCMV-specific T lymphocytes in whole blood in patients after stem cell transplantation. J Immunol Methods 311:164-173

6. Chang WL, Tarantal AF, Zhou SS, Borowsky AD, Barry PA (2002) A recombinant rhesus cytomegalovirus expressing enhanced green fluorescent protein retains the wild-type phenotype and pathogenicity in fetal macaques. J Virol 76:9493-9504

7. Drexler I, Staib C, Sutter G (2004) Modified vaccinia virus Ankara as antigen delivery system: how can we best use its potential? Curr Opin Biotechnol 15:506-512

8. Fowler KB, Stagno S, Pass RF (2003) Maternal immunity and prevention of congenital cytomegalovirus infection. JAMA 289:1008-1011

9. Fowler KB, Stagno S, Pass RF, Britt WJ, Boll TJ, Alford CA (1992) The outcome of congenital cytomegalovirus infection in relation to maternal antibody status. N Engl J Med 326:663-667

10. Gonczol E, Berensci K, Pincus S, Endresz V, Meric C, Paoletti E, Plotkin SA (1995) Preclinical evaluation of an ALVAC (canarypox)—human cytomegalovirus glycoprotein B vaccine candidate. Vaccine 13:1080-1085

11. Hansen SG, Strelow LI, Franchi DC, Anders DG, Wong SW (2003) Complete sequence and genomic analysis of rhesus cytomegalovirus. J Virol 77:6620-6636

12. Huff JL, Eberle R, Capitanio J, Zhou SS, Barry PA (2003) Differential detection of $\mathrm{B}$ virus and rhesus cytomegalovirus in rhesus macaques. J Gen Virol 84:83-92
13. Loomis-Huff JE, Eberle R, Lockridge KM, Rhodes G, Barry PA (2001) Immunogenicity of a DNA vaccine against herpes B virus in mice and rhesus macaques. Vaccine 19:4865-4873

14. Nigro G, Adler SP, La Torre R, Best AM (2005) Passive immunization during pregnancy for congenital cytomegalovirus infection. N Engl J Med 353:1350-1362

15. Pass RF, Duliege AM, Boppana S, Sekulovich R, Percell S, Britt W, Burke RL (1999) A subunit cytomegalovirus vaccine based on recombinant envelope glycoprotein B and a new adjuvant. J Infect Dis 180:970-975

16. Robinson HL, Montefiori DC, Villinger F, Robinson JE, Sharma S, Wyatt LS, Earl PL, McClure HM, Moss B, Amara RR (2006) Studies on GM-CSF DNA as an adjuvant for neutralizing Ab elicited by a DNA/MVA immunodeficiency virus vaccine. Virology 352:285-294

17. Schleiss MR, Bourne N, Bernstein DI (2003) Preconception vaccination with a glycoprotein $\mathrm{B}(\mathrm{gB})$ DNA vaccine protects against cytomegalovirus (CMV) transmission in the guinea pig model of congenital CMV infection. J Infect Dis 188:1868-1874

18. Schleiss MR, Bourne N, Stroup G, Bravo FJ, Jensen NJ, Bernstein DI (2004) Protection against congenital cytomegalovirus infection and disease in guinea pigs, conferred by a purified recombinant glycoprotein B vaccine. J Infect Dis 189:1374-1381

19. Stratton KR, Durch JS, Lawrence RS (2000) Vaccines for the 21st century: a tool for decisionmaking. National Academy Press, Washington, DC

20. Wang Z, La Rosa C, Lacey SF, Maas R, Mekhoubad S, Britt WJ, Diamond DJ (2006) Attenuated poxvirus expressing three immunodominant CMV antigens as a vaccine strategy for CMV infection. J Clin Virol 35:324-331

21. Wang Z, La Rosa C, Li Z, Ly H, Krishnan A, Martinez J, Britt WJ, Diamond DJ (2007) Vaccine properties of a novel marker genefree recombinant modified vaccinia Ankara expressing immunodominant CMV antigens pp65 and IE1. Vaccine 25:1132-1141

22. Wang Z, La Rosa C, Maas R, Ly H, Brewer J, Mekhoubad S, Daftarian P, Longmate J, Britt WJ, Diamond DJ (2004) Recombinant modified vaccinia virus Ankara expressing a soluble form of glycoprotein B causes durable immunity and neutralizing antibodies against multiple strains of human cytomegalovirus. J Virol 78:3965-3976

23. Wyatt LS, Belyakov IM, Earl PL, Berzofsky JA, Moss B (2008) Enhanced cell surface expression, immunogenicity and genetic stability resulting from a spontaneous truncation of HIV Env expressed by a recombinant MVA. Virology (in press)

24. Yeager AS, Grumet FC, Hafleigh EB, Arvin AM, Bradley JS, Prober CG (1981) Prevention of transfusion-acquired cytomegalovirus infections in newborn infants. J Pediatr 98:281-287

25. Yue Y, Kaur A, Eberhardt MK, Kassis N, Zhou SS, Tarantal AF, Barry PA (2007) Immunogenicity and protective efficacy of DNA vaccines expressing rhesus cytomegalovirus glycoprotein $\mathrm{B}$, phosphoprotein $65-2$, and viral interleukin-10 in rhesus macaques. J Virol 81:1095-1109

26. Yue Y, Kaur A, Zhou SS, Barry PA (2006) Characterization and immunological analysis of the rhesus cytomegalovirus homologue (Rh112) of the human cytomegalovirus UL83 lower matrix phosphoprotein (pp65). J Gen Virol 87:777-787

27. Yue Y, Zhou SS, Barry PA (2003) Antibody responses to rhesus cytomegalovirus glycoprotein B in naturally infected rhesus macaques. J Gen Virol 84:3371-3379

28. Zhong J, Khanna R (2007) Vaccine strategies against human cytomegalovirus infection. Expert Rev Anti Infect Ther 5:449-459 